\section{AB0801 EFFECTS OF GUSELKUMAB, A MONOCLONAL ANTIBODY THAT SPECIFICALLY BINDS TO THE P19- SUBUNIT OF INTERLEUKIN-23, ON DACTYLITIS AND ENTHESITIS IN PATIENTS WITH ACTIVE PSORIATIC ARTHRITIS: POOLED RESULTS THROUGH WEEK 24 FROM TWO PHASE 3 STUDIES}

D. Mcgonagle ${ }^{1}$, I. Mcinnes ${ }^{2}$, A. Deodhar ${ }^{3}$, G. Schett ${ }^{4}$, P. J. Mease ${ }^{5}$, M. Shawi ${ }^{6}$,

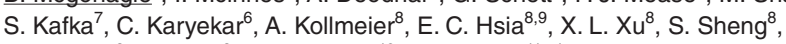
P. Agarwal ${ }^{8}$, B. Zhou ${ }^{8}$, C. T. Ritchlin ${ }^{10}$, P. Rahman ${ }^{11} .{ }^{1}$ Leeds Biomed Res Ctr, $U$ Leeds, Leeds, United Kingdom; ${ }^{2} U$ Glasgow, Glasgow, United Kingdom; ${ }^{3}$ Oregon Health \& Science U, Portland, United States of America; ${ }^{4} F A U$, Erlangen-Nürnburg, Germany; ${ }^{5}$ Swedish Med Ctrl Providence St Joseph Health and U Wash School of Med, Seattle, United States of America; ${ }^{6}$ Janssen Global Svcs, LLC, Horsham, United States of America; ${ }^{7} J a n s s e n ~ S c i e n t i f i c$ Affairs, LLC, Horsham, United States of America; ${ }^{8} \mathrm{Janssen}$ Research \& Development, LLC, Spring House, United States of America; ${ }^{9} U$ Penn Med Ctr, Philadelphia, United States of America; ${ }^{10} \mathrm{U}$ Rochester Med Center, Rochester, United States of America; ${ }^{11}$ Memorial U Newfoundland, St. Johns, Canada

Background: Guselkumab (GUS), a novel monoclonal antibody that specifically binds to the p19-subunit of IL-23, demonstrated efficacy in the Ph 3 DISCOVER-1 (D1) \& DISCOVER-2 (D2) trials of pts with active psoriatic arthritis (PsA) ${ }^{1,2}$ Dactylitis \& enthesitis, key PsA clinical manifestations, can be difficult to treat and may portend more significant disease burden. ${ }^{3,4}$

Objectives: In pts with dactylitis or enthesitis at baseline, assess: 1) changes in symptoms over time and 2) relationships between improvements in dactylitis or enthesitis and other PsA domains.

Methods: Adults with active PsA despite standard therapies were eligible for D1 \& D2. Approx. $30 \%$ of D1 pts previously received 1-2 TNF inhibitors; D2 pts were biologic-naïve. Pts were randomized 1:1:1 to GUS 100mg Q4W; GUS 100mg at W0, W4, Q8W; or PBO. Independent assessors evaluated dactylitis (total score: $0-60)$ \& enthesitis (Leeds Enthesitis Index [LEI]; total score 0-6). Dactylitis and enthesitis findings through W24 were prespecified to be pooled across D1 \& D2. P-values are unadjusted. We assessed changes in dactylitis and LEI scores over time (ANCOVA); associations between dactylitis or enthesitis resolution and ACR/PASI responses at W24 (Chi-square); and correlations between dactylitis or LEI and HAQ-DI/SF-36 change scores at W24 (Spearman's correlation). AEs through W24 were reported. ${ }^{1,2}$

Results: At W0, $42 \%$ of pooled D1+D2 pts had dactylitis; $65 \%$ had enthesitis. GUS improved dactylitis and LEI scores vs PBO at W8, W16, W24. GUS vs PBO differences were significant for dactylitis changes at W16 \& W24 and LEI changes at W8 (Q4W only), W16 \& W24; no dose response was observed (Fig). Rates of dactylitis or enthesitis resolution by W24 were consistently significantly $(p<0.001)$ associated with ACR20/50/70 and PASI75/90 response (Table). In GUS-treated pts at W24, significant correlations were observed between dactylitis change scores and PASI ( $p<0.001$ Q4W; $p=0.006$ Q8W) and SF-36 MCS $(p=0.038 \mathrm{Q} 4 \mathrm{~W} ; \mathrm{p}=0.003 \mathrm{Q} 8 \mathrm{~W})$ changes, and between LEI and HAQ-DI change scores ( $<<0.001 \mathrm{Q} 4 \mathrm{~W} ; \mathrm{p}=0.005 \mathrm{Q} 8 \mathrm{~W})$. No consistent correlations/associations were observed between dactylitis or LEI scores and other clinical outcomes.

Conclusion: In PsA pts with dactylitis or enthesitis at WO, GUS improved dactylitis or LEI scores vs PBO by W8; treatment differences were significant at W16 \& W24. Resolution of dactylitis or enthesitis was significantly associated with clinically meaningful improvements in PsA joint \& skin symptoms. Improved dactylitis scores correlated with improved skin symptoms and mental health; improved LEI scores correlated with improved physical function.

References:

[1] Deodhar A (A\#807),

[2] Mease P (A\#L13), Arthritis Rheumatol 2019;71(suppl 10);

[3] DOI: 10.1186/s13075-017-1399-5; ${ }^{4} \mathrm{DOI}: 10.1016 / \mathrm{j} . \mathrm{semarthrit} .2018 .02 .002$

Table. Pooled DISCOVER-1\&2: associations between dactylitis/enthesitis resolution and joint/skin response

\begin{tabular}{|c|c|c|c|c|c|c|c|}
\hline & & ACR20 & ACR50 & ACR70 & & PASI $75^{a}$ & PASI90 $^{\text {a }}$ \\
\hline Dactylitis resolution ${ }^{b}$ & $\mathrm{~N}$ & $\% p t s$ & $\%$ pts & $\%$ pts & $\mathbf{N}$ & $\%$ pts & $\%$ pts \\
\hline Q4W & 373 & $55^{\star}$ & $34^{*}$ & $16^{*}$ & 121 & $78^{*}$ & $55^{\star}$ \\
\hline Q8W & 375 & $53^{*}$ & $31^{*}$ & $16^{\star}$ & 116 & $80^{*}$ & $65^{\star}$ \\
\hline PBO & 372 & $26^{\star}$ & $12^{\star}$ & $5^{\star}$ & 115 & $19^{\star}$ & $10^{*}$ \\
\hline \multicolumn{8}{|l|}{ Enthesitis resolution $^{c}$} \\
\hline Q4W & 243 & $34^{*}$ & $31^{*}$ & $11^{*}$ & 187 & $82^{*}$ & $63^{*}$ \\
\hline Q8W & 230 & $40^{\star}$ & $7^{*}$ & $12^{*}$ & 162 & $77^{\star}$ & $62^{*}$ \\
\hline PBO & 255 & $14^{*}$ & $13^{*}$ & $5^{*}$ & 182 & $19^{*}$ & $9^{*}$ \\
\hline
\end{tabular}

${ }^{*} p<0.001$ (Chi-square)

a in with $\geq 3 \%$ BSA psoriasis \& IGA $\geq 2$ at WO

${ }^{b}$ In pts with D at WO

${ }^{c}$ In pts with E at WO
Fig. Pooled DISCOVER-1\&2 LS mean changes from baseline over time in A) dactylitis and B) LEI scores

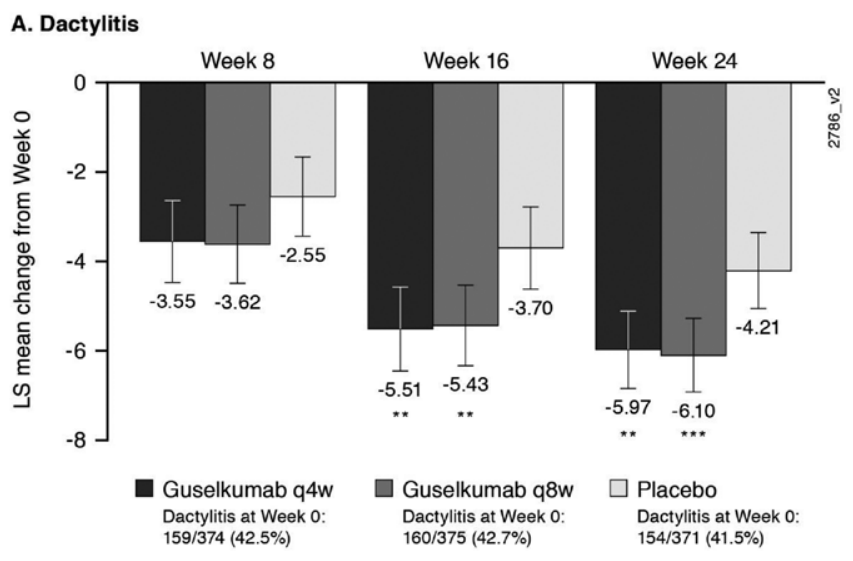

B. Enthesitis

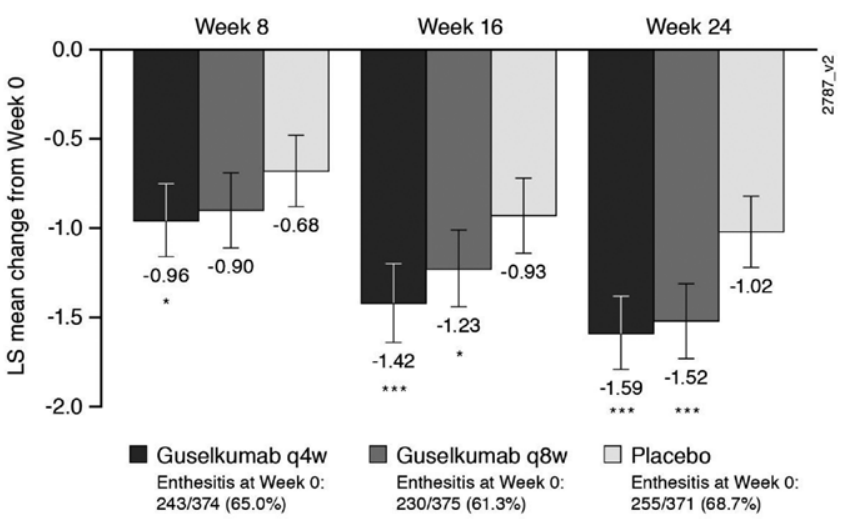

$\because, \cdots, \cdots$ Unadjusted (nominal) $\mathrm{p}<0.05,0.01,0.001$ vs placebo (not controlled for multiplicity; interpret only as supportive)

\section{Acknowledgments: None}

Disclosure of Interests: Dennis McGonagle Grant/research support from: Janssen Research \& Development, LLC, lain McInnes Grant/research support from: Bristol-Myers Squibb, Celgene, Eli Lilly and Company, Janssen, and UCB, Consultant of: AbbVie, Bristol-Myers Squibb, Celgene, Eli Lilly and Company, Gilead, Janssen, Novartis, Pfizer, and UCB, Atul Deodhar Grant/research support from: AbbVie, Eli Lilly, GSK, Novartis, Pfizer, UCB, Consultant of: AbbVie, Amgen, Boehringer Ingelheim, Bristol Myer Squibb (BMS), Eli Lilly, GSK, Janssen, Novartis, Pfizer, UCB, Speakers bureau: AbbVie, Amgen, Boehringer Ingelheim, Bristol Myer Squibb (BMS), Eli Lilly, GSK, Janssen, Novartis, Pfizer, UCB, Georg Schett Speakers bureau: AbbVie, BMS, Celgene, Janssen, Eli Lilly, Novartis, Roche and UCB, Philip J Mease Grant/research support from: Abbott, Amgen, Biogen Idec, BMS, Celgene Corporation, Eli Lilly, Novartis, Pfizer, Sun Pharmaceutical, UCB - grant/research support, Consultant of: Abbott, Amgen, Biogen Idec, BMS, Celgene Corporation, Eli Lilly, Novartis, Pfizer, Sun Pharmaceutical, UCB - consultant, Speakers bureau: Abbott, Amgen, Biogen Idec, BMS, Eli Lilly, Genentech, Janssen, Pfizer, UCB - speakers bureau, May Shawi Shareholder of: Johnson \& Johnson, Employee of: Janssen Research \& Development, LLC, Shelly Kafka Employee of: Janssen Scientific Affairs, LLC, Chetan Karyekar Shareholder of: Johnson \& Johnson, Consultant of: Janssen, Employee of: Janssen Global Services, LLC. Previously, Novartis, Bristol-Myers Squibb, and Abbott Labs., Alexa Kollmeier Shareholder of: Johnson \& Johnson, Employee of: Janssen Research \& Development, LLC, Elizabeth C Hsia Shareholder of: Johnson \& Johnson, Employee of: Janssen Research \& Development, LLC, Xie L Xu Shareholder of: Johnson \& Johnson, Employee of: Janssen Research \& Development, LLC, Shihong Sheng Shareholder of: Johnson \& Johnson, Employee of: Janssen Research \& Development, LLC, Prasheen Agarwal Shareholder of: Johnson \& Johnson, Employee of: Janssen Research \& Development, LLC, Bei Zhou Shareholder of: Johnson \& Johnson, Employee of: Janssen Research \& Development, LLC, Christopher T. Ritchlin Grant/research support from: UCB Pharma, AbbVie, Amgen, Consultant of: UCB Pharma, Amgen, AbbVie, Lilly, Pfizer, Novartis, Gilead, Janssen, Proton Rahman Grant/research support from: Janssen and Novartis, Consultant of: Abbott, AbbVie, Amgen, BMS, Celgene, Lilly, Janssen, Novartis, and Pfizer., Speakers bureau: Abbott, AbbVie, Amgen, BMS, Celgene, Lilly, Janssen, Novartis, Pfizer DOI: 10.1136/annrheumdis-2020-eular.836 